However, in the increase of the electron density of the bacilli stained by Ziehl-Neelsen's method, other factors, such as the stain and the lipid material, should be taken into consideration.

In short, in the staining procedures of ZiehlNeelsen's and Fontes' methods, most of the cells of $B C G$ contract and their electron density increases though the electron-dense granules disappear. Accordingly, the granules detected in the specimens stained by these methods are different from the electron-dense granules, which are considered to occur naturally. 'The carbol of the stains which are used is among the causes of these changes.

We thank Profs. Hibi and Kuroya, of Tôhoku University, for their assistance, and Dr. E. M. Brieger, Strangeways Research Laboratory, Cambridge, for his instructive comments.

$$
\begin{aligned}
& \text { T. Ebina } \\
& \text { C. Shinohara } \\
& \text { H. SaIto } \\
& \text { K. FukUshi } \\
& \text { J. SUzuki }
\end{aligned}
$$

Research Institute for Tuberculosis and Leprosy, Tôhoku University, Sendai. March 23.

${ }^{2}$ Takeya, K., Koike, M., Uchida, T., Inoue, S., and Nomiyama, K., J. Electron Micr., 2, 29 (1954).

${ }^{2}$ Glauert, A. M., and Brieger, E. M., J. Gen. Microbiol., 13, 310 (1955).

\section{Lysis of Vibrio cholerae by Thymol}

CULTuRes of Vibrio cholerae are very sensitive to thymol. The addition of $50 \mathrm{mgm}$. of this substance to $10 \mathrm{ml}$. of $4-\mathrm{hr}$. broth cultures results in rapid clearing due to massive lysis of the organisms. 'This takes about $30-45 \mathrm{~min}$. at $37^{\circ} \mathrm{C}$, by which time the cultures are sterile. As thymol is soluble in aqueous media only to the extent of 1 in 1,500 , the effective concentration required to lyse $V$. cholerae may be of this order. The rapidity with which this occurs simulates the phenomenon of 'induction' observed in certain lysogenic bacteria, when they are exposed to ultra-violet radiation or X-rays or treated with chemical mutagens. However, lysogenic cultures of $V$. cholerae exposed to thymol do not reveal any appreciable increase in free phage particles in the lysates. Further, 'indicator' strains which have proved useful in the detection of lysogenic strains and which appear to be non-lysogenic themselves are lysed equally effectively by thymol.

Other phenols such as catechol, resorcinol, menthol and carbolic acid do not lyse these cultures to a perceptible degree in such a short time. Of these, only carbolic acid is lethal to $V$. cholerae. It is possible that thymol, by virtue of its low toxicity and marked vibriolytic activity, may be useful in clinical and laboratory studies on cholera.

Central Drug Research Institute,

K. BHASKARAN

Lucknow. March 1.

\section{Fomes lignosus, a Pyrimidine-deficient Fungus}

I'He growth requirements of a number of brownroot and white-root disease fungi have already been studied. It is generally recognized that the majority of the Hymenomycetes are partially or totally deficient in thiamine (aneurin, vitamin $\left.B_{1}\right)^{1}$.

so far as can be ascertained from the literature available, one of the most important root disease
Table 1. $p \mathrm{H}$ AND MYCELICM (DRY WEIGHT IN MaM.) OF Fomes Tignosus ON K YOP-GLdCOSE MEDIUM WITH AND WITHODT ASPARAGINE,

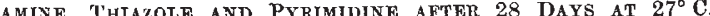

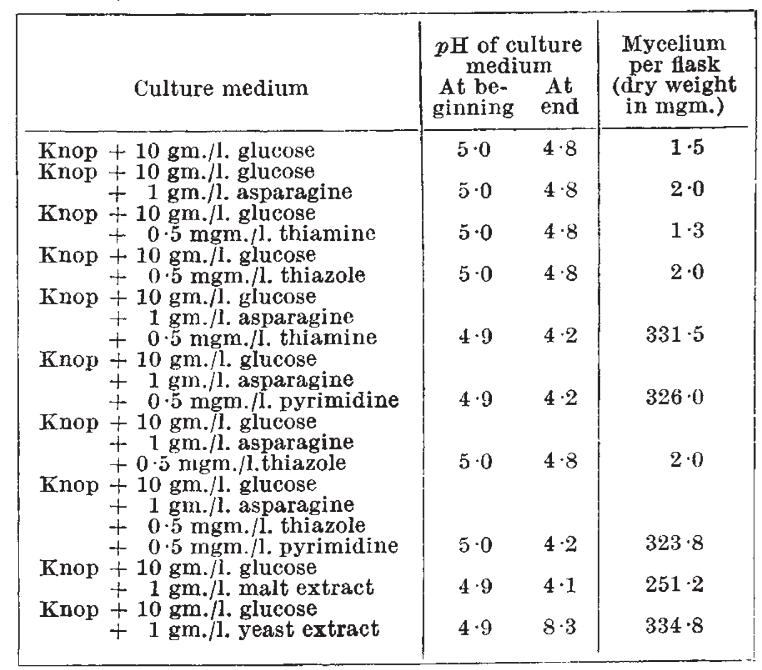

fungi, especially of tropical plants, Fomes lignosus (Klot.) Heim ex. Pat., has not so far been investigated in this respect ${ }^{2}$. Attempts to grow Fomes lignosus on artificial media, as those of Czapek, Fries, Richard and $\mathrm{Knop}^{3}$, have been unsuccessful. However, as soon as organic materials such as malt extract, yeast extract or decoctions of fresh rubber wood or of rubber leaves were added, luxuriant growth of the fungus occurred, and, in the culture flasks, the wellknown rhizomorphs were formed.

'The experiments were carried out in $500-\mathrm{ml}$. 'Jena' Erlenmeyer flasks. Each series consisted of ten flasks, and each flask contained $100 \mathrm{ml}$. of the liquid culture medium. All experiments were replicated three times. "The chemicals used were of the "pro analysis" grade (Merck) or the special "Biochemicals" of Hoffmann-La-Roche.

To obtain a uniform inoculum for these experiments the fungus was grown in advance for 42 days on pieces of fresh rubber wood, and a mycelium suspension was prepared by adding $100 \mathrm{ml}$. of sterile distilled water and vigorously shaking the culture flasks. Preculturing the fungus on small pieces of wood was advantageous compared with preculturing on a rice or liquid medium. The Erlenmeyer flasks were inoculated by the wet method, each flask with $0.5 \mathrm{ml}$. of the mycelium suspension, and the cultures were incubated at $27^{\circ} \mathrm{C}$. Knop solution containing $10 \mathrm{gm}$./l. of vitamin-free glucose was used as basic medium for these experiments. The results of the experiments are presented in Table 1.

Fomes lignosus is a fungus which is unable to synthesize the whole thiamine (thiamine hydrochloride, $\mathrm{C}_{12} \mathrm{H}_{17} \mathrm{ON}_{4} \mathrm{SCl} . \mathrm{HCl}$ ) molecule. It can synthesize only one part, thiazole (4-methyl-5( $\beta$-oxyethyl)-thiazole, $\left.\mathrm{C}_{8} \mathrm{H}_{9} \mathrm{ONS}\right)$; the other part, pyrimidine (2-methyl-4-amino-5-aminomethyl-pyrimidine-dihydrochloride, $\mathrm{C}_{6} \mathrm{H}_{10} \mathrm{~N}_{4} .2 \mathrm{HCl}$ ), must be supplied from other sources.

A further interesting fact brought out in these experiments is that the pure Knop solution with $10 \mathrm{gm} . / \mathrm{l}$. glucose and thiamine or pyrimidine is not sufficient for satisfactory growth and that in addition asparagine had to be added. How far asparagine can be replaced by other organic nitrogen sources and 